\title{
Tomografía de la red en un IXP: Primer análisis del PIT Bolivia
}

\author{
Esteban Carisimo \\ INTECIN (UBA-CONICET) \\ Facultad de Ingeniería \\ Universidad de Buenos Aires \\ Ciudad de Buenos Aires, Argentina \\ Email: carisimo@cnet.fi.uba.ar \\ Recibido: 12/10/16; Aceptado: 06/07/17
}

\begin{abstract}
Internet Exchange Points (IXPs) have been responsible for leading the most significant change on Internet ecosystem in the last 15 years. Despite the popularity of IXPs, a large number of countries delayed to introduce this infrastructure in their national networks. In 2013, Bolivia encouraged the creating of a national IXP, which motivated us to make the first study about an IXP in Latin America, in a country which had not deployed this infrastructure yet. However, due to the lack of vantage points of measurement projects in Bolivia, it was necessary to develop and deploy a measurement platform, which was called PladMeD, to be able to analyze the influence of the IXP. This works presents a six-month analysis about the influence of the IXP on the national network and it mainly focuses on the modifications in the interconnection as well as the quality of service.

Resumen- Los Puntos de Intercambios de Tráfico (PIT) han sido protagonistas fundamentales del mayor cambio del ecosistema de Internet en los últimos 15 años. A pesar de la popularidad de los PITs, gran cantidad de países demoraron en incorporar esta infraestructura a sus redes. En 2013, Bolivia impulsó la creación del PIT nacional, lo que motivó nuestro interés de estudiar por primera vez un PIT en la región, en un país que aún no contara con esta infraestructura. Sin embargo, ante la ausencia de sondas de medición de Internet en Bolivia fue necesario desarrollar una plataforma (PladMeD) para observar la influencia del PIT. En este artículo se estudió durante seis meses el efecto del PIT en la red nacional, enfocándose en las modificaciones en la interconexión y la calidad de servicio en la red nacional.
\end{abstract}

\section{INTRODUCCIÓN}

El gobierno boliviano promulgó la Ley de Telecomunicaciones en $2011^{1}$, por la cual promovía la creación del primer Punto de Intercambio de Tráfico (PIT, del inglés Internet Exchange Point, IXP) para interconectar a los seis mayores proveedores del país (AXS, COTAS, ENTEL, COMTECO, NUEVATEL y TELECEL). Este hecho que motivó nuestro interés en investigar el impacto de la creación del PIT en una red nacional, la cual no contaba con ningún PIT y además contaba con escasa interconexión. Sin embargo, debido al proceso de propagación de rutas de BGP y la carencia de puntos de observación en Bolivia, el conocimiento de la red y los efectos de PIT serían imperceptibles desde el exterior, lo cual nos condujo al despliegue de una plataforma de medición local, capaz de permitirnos observar estos fenómenos.

\footnotetext{
${ }^{1}$ La Resolución Administrativa Regulatoria ATT-DJ-RA TL 0482/2013 establece la creación del PIT. Esta se basa en resoluciones, decretos y leyes previamente promulgados.
}

Un PIT es una arquitectura de Internet que tiene como finalidad poder generar múltiples interconexiones entre Sistemas Autónomos (ASes, del inglés Autonomous System, AS) en un único punto. Más aún, su creación y funcionamiento se encuentran fundamentados bajo el leitmotive "mantener local el tráfico local", ya que los PITs buscan dotar de interconexión local a los ASes, con el objetivo que el tráfico intercambiado entre éstos no requiera el uso de la infraestructura nacional o internacional.

Los vínculos entre Sistemas Autónomos pueden ser clasificados bajo dos categorías dependiendo de los acuerdos comerciales entre ambos participantes. La primer categoría se denomina cliente-proveedor, y se observa cuando el acuerdo de interconexión entre ASes está sujeto al cobro. De manera opuesta, la categoría vínculos entre pares se denomina a aquellas inteconexiones en las cuales ninguno del los dos ASes involucrados le exige una tarifa a la otra parte interesada. En el caso de una interconexión clienteproveedor, el cliente le abona una tarifa la proveedor a cambio de que este le provea un mejor acceso e interconexión a la red. Para los vínculos entre pares, ambos ASes deciden establecer un vínculo dedicado de manera tal que los usuarios de cada AS puedan acceder al otro AS por medio de este enlace. A su vez, dado el espíritu de beneficio mutuo, los vínculos entre pares únicamente brindarán acceso al AS conectado y no así al resto de la red. Dado que los vínculos cliente-proveedor proveerán un mejor acceso a la red para el cliente, el tráfico será marcadamente asimétrico, mientras que el los vínculos entre pares dado que el beneficio es recíproco, el tráfico será simétrico. Aunque los enlaces entre pares presenten una mejora significativa en la interconexión de los ASes, sólo se justifican en el caso en el que el volumen de tráfico entre ambos ASes represente una gran proporción del tráfico total, ya que de otra manera no se justificaría el costo de contratar la infraestructura de capa física para generar la interconexión. Este motivo económico genera que en Internet haya una escasa cantidad de vínculos entre pares.

A raíz de que en la mayoría de las circunstancias los aspectos económicos no justifican vínculos entre pares, los PITs buscan hacer extensiva esta propiedad a múltiples Sistemas Autónomos. La implementación técnica simplemente requiere que un AS contrate un vínculo físico hacia el PIT, y luego allí podrá generar interconexión con todos los otros ASes participantes del PIT. De esta manera, el PIT permite reducir drásticamente la cantidad enlaces necesarios 


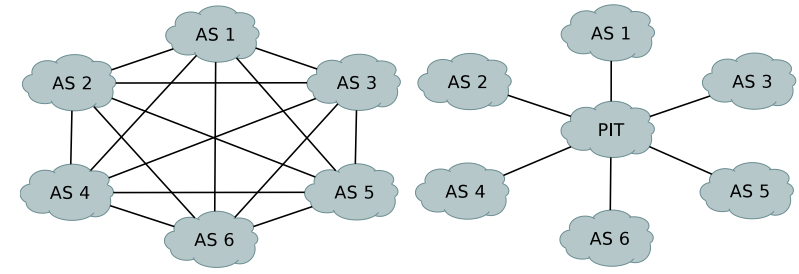

Fig. 1. Comparación de cantidad de enlaces necesario para generar una interconexión completa (cliqué) utilizando vínculos entre socios (izquierda) o un PIT (derecha). La Figura refleja como el PIT reduce orden del número de enlaces de $\mathcal{O}\left(n^{2}\right)$ a $\mathcal{O}(n)$.

para interconectar $n$ Sistemas Autónomos, llevándola de $\frac{1}{2} \cdot n \cdot(n-1)$ en el vínculos entre pares a $n$ en el caso del PIT, tal como se observa en la Figura 1.

El hecho de que un AS tenga presencia en un PIT que reúna varios ASes locales, genera que cada uno de los ASes participante pueda reducir el tráfico hacia sus proveedores, generando reducción en la congestión y en los costos. Este aspecto fue sumamente relevante a principios de la década 1990, ya que los proveedores europeos promovieron la creación de varios PITs, de manera tal de reducir el tráfico por los enlaces submarinos hacia Estados Unidos, donde se encontraba en aquel entonces el backbone de la red [1]. Los proveedores observaron que la mayor parte del tráfico tenía origen y destino local, pero no obstante era enviado a través de costosos enlaces submarinos para luego retornar a Europa. Este auge llevó a la creación de LINX (Londres), AMS-IX (Ámsterdam) y DE-CIX (Alemania), que en la actualidad se encuentran entre los PITs con mayor tráfico diario en el mundo. A su vez, durante el mismo período, el proceso de transformación de Internet a través de su apertura comercial, llevó que la privatización de la NSFNET ${ }^{2}$ diera lugar a la creación de cuatro PITs para los grandes centros urbanos en Estados Unidos, ubicados en Washington D.C., Nueva York/New Jersey, Chicago y California. Las virtudes de los PIT ha llevado a que en la actualidad se estimen en 376 alrededor del mundo, donde 95 países ya han implementando al menos un PIT [2].

Sin embargo, las ventajas de un PIT no son únicamente comerciales sino que también brindan mejoras significativas en la calidad de Internet. En primer lugar, si los paquetes son distribuidos sin abandonar la región, se observará una marcada reducción en la latencia, producto de una menor distancia física recorrida. Un ejemplo es el anterior caso mencionado de Europa, donde los IXPs conectaron ASes que se encontraban a unos pocos kilómetros de distancia. Esta optimización produjo que los paquetes no deban recorrer miles de kilómetros de cables submarinos para alcanzar Estados Unidos y luego retornar. Más aún, en la estructura de Internet actual los PIT se han convertido en actores fundamentales para facilitar el acceso al contenido. Durante los últimos años, la mayor cantidad de tráfico de Internet se ha concentrado en cantidad muy reducida de servicios, tales como Google, YouTube, Netflix, Facebook y otras redes sociales, que generalmente suelen ser contenidos de origen extranjero. Sin embargo, para reducir la latencia y mejorar la calidad de experiencia de usuarios, estos servicios son

${ }^{2}$ NSFNET: National Science Foundation Network, red primigenia de Internet. servidos por medio de redes de distribución de contenido (CDN, del inglés Content Delivery Network) [3], las cuales buscan ubicar servidores replicados lo más próximo posible a los usuarios. Entonces, los PIT y las CDN encuentran un beneficio mutuo, ya que los usuarios en los ASes del PIT podrán acceder al contenido localmente, los ASes podrán reducir su tráfico internacional hacia los servicios brindados por las CDNs y las CDNs podrán cubrir con un único punto de presencia a gran cantidad de usuarios. Aunque las CDNs suelen tener vínculos directos con los grandes proveedores de Internet (del inglés Internet Service Providers, ISP) de cada país, la presencia en PITs hace que los pequeños ISPs puedan acceder localmente al contenido, ya que ellos nunca hubieran podido lograr un acuerdo para generar un vínculos entre pares con la $\mathrm{CDN}$.

\section{LITERATURA RELACIONADA}

Los PITs han sido vastamente estudiados por la comunidad científica en los últimos años, principalmente debido a su rápida proliferación a nivel mundial. En particular, uno de los principales puntos de estudio ha sido su influencia dentro del desjerarquización que ha estado sucediendo la red por al menos los últimos 10 años [4], [5].

Uno de los artículos iniciadores de la investigación de PITs fue Xu et al. [6] que en 2004 se enfocó en descubrir la mayor cantidad de PITs posibles, para luego poder analizar su influencia en la interacción de los ASes. Dado que no es posible identificar un PIT a menos que se cuente con un punto de observación colocado en un Sistema Autónomo miembro del PIT, Xu et al. combinó multiples metodologías y fuentes de datos, tales como traceroutes (Skitter-CAIDA [7], Scriptroute [8]), tablas BGP (Route Views [9], RIPE RIS [10]) y bases de datos públicas (Packet Clearing House $(\mathrm{PCH})$ ). A través de esta extensa y variada cantidad de datos, los autores pudieron caracterizar 82 de los 148 PITs activos en todo el mundo en 2004.

Retomando el trabajo de Xu et al., Augustin et al. [11] buscó develar la mayor cantidad de vínculos ocultos en PITs, con el objetivo de enriquecer la topología de Internet. En este sentido, el artículo utilizó las mismas fuentes de información que su antecesor, pero agregó los Looking Glasses ${ }^{3}$ (LGs) como punto de observación. Valiéndose de que gran cantidad de PITs han instalado LGs, los autores los tomaron para recolectar las tablas BGP, cuando esto fuera posible, o como fuente de traceroutes o pings. Luego de las exploraciones los resultados fueron 223 PITs relevados, agregando 44k nuevas interacciones entre ASes en estos PITs.

Sin embargo, la investigación no ha estado únicamente centrada en develar la influencia global de los PITs, sino que también han surgido trabajos enfocados en PITs de una región o simplemente en un PIT específico. Entre los más destacados se encuentran Ager et al. [12], Cardona et al. [13], Gupta et al. [14] y Fanou et al. [15]. Europa cuenta con gran cantidad de PITs, Ager et al. y Cardona et al. en este continente son dos publicaciones que han estudiado casos específicos de PITs. En cuanto, Gupta et al. y Fanou

${ }^{3}$ Looking Glasses: Servidores de acceso público vía web los cuales permiten ejecutar ciertos comandos, tales como pings y traceroutes, desde su plataforma hacia algún destino. Se utilizó como herramientas de diagnóstico. 
et al. se han enfocado en el estudio de la influencia de los PITs en África.

Europa no solamente cuenta con una gran cantidad de PITs sino que también con aquellos de mayor cantidad de tráfico diario. Resguardando la anonimidad del PIT, Ager et al. ha estudiado uno de los PIT de mayor tráfico de Europa utilizando NetFlow ${ }^{4}$, logrando caracterizar la cantidad de miembros, sus ubicaciones y sus perfiles de tráfico. Enfocándose en un PIT de menor envergadura, Cardona et al. ha mostrado la evolución del PIT de Eslovaquia (SIX ${ }^{5}$ ) dejando en evidencia que una vez que el PIT logra una cantidad considerable de tráfico, gran cantidad de nuevos ASes se incorporan al mismo.

La interconexión y la distribución de contenidos presenta patrones sumamente diferentes entre países desarrollado y en vías de desarrollo. Gupta et al. y Fanou et al. han estudiado la influencia de los PITs en África, y las limitaciones que aún existen en gran cantidad de países de ese continente por más que cuenten con PITs. Utilizando múltiples fuentes de información sumado el proyecto BISmark ${ }^{6}$ [16], Gupta et al. reveló como gran cantidad de usuarios en redes africanas perciben gran latencia a causa de que los ISPs suelen estar interconectados a través de LINX. De la misma manera, Fanou et al. utilizando RIPE Atlas ${ }^{7}$ [17], destacó la influencia de proveedores de tránsito franceses en los países francófonos y de proveedores británicos en países anglófonos.

En cuanto a los trabajos de investigación en Latinoamérica, Silva Berenguer et al. [18] ha recientemente analizado la ausencia de conocimiento acerca de las redes de la región en los proyectos de investigación de escala global. Utilizando información de los PITs de Brasil (PTT), Argentina (CABASE) y los resultados obtenidos de este artículo, se han podido cuantificar las diferencias que se pueden obtener en la topología de Internet al agregar esta información.

El proceso de desjarquización de la red descripto en Dhamdhere et al. [4] y Labovitz et al. [5] puede ser sinteticamente resumido en los resultados presentados por Gill et al. [19] en 2008. De acuerdo con lo que publicó este último artículo, $60 \%$ de las rutas analizadas entre usuarios y los tres proveedores de contenidos más relevantes de ese momento (Microsoft, Yahoo!, Google), no contenía ningún TIER-1. En estos tres artículos se subraya, la sinergia entre PITs y CDNs para lograr estos resultados.

\section{Metodología}

Ante el interés de relevar el recientemente PIT instalado en Bolivia ${ }^{8}$, a mediados de 2014 se comenzó a estudiar cuales eran los recursos necesarios para poder llevarlo a cabo. Recordando que el leitmotive de los PITs "mantener

\footnotetext{
${ }^{4}$ NetFlow es un protocolo desarrollado por Cisco para poder capturar y procesar el tráfico en los dispositivos intermedios, tales como routers o switches.

${ }^{5}$ SIX: Slovak Internet Exchange. http://www.six.sk

${ }^{6}$ BISmark proyecto de medición distribuido desarrollado sobre routers comerciales en end-hosts.

${ }^{7}$ RIPE Atlas es un proyecto de medición distribuido a lo largo del mundo que cuenta con cerca de 10.000 puntos de obervación.

${ }^{8}$ El PIT de Bolivia comenzó a operar el 13 de noviembre de 2013. http://www.pit.bo/index.php/preguntas-frecuentes
}

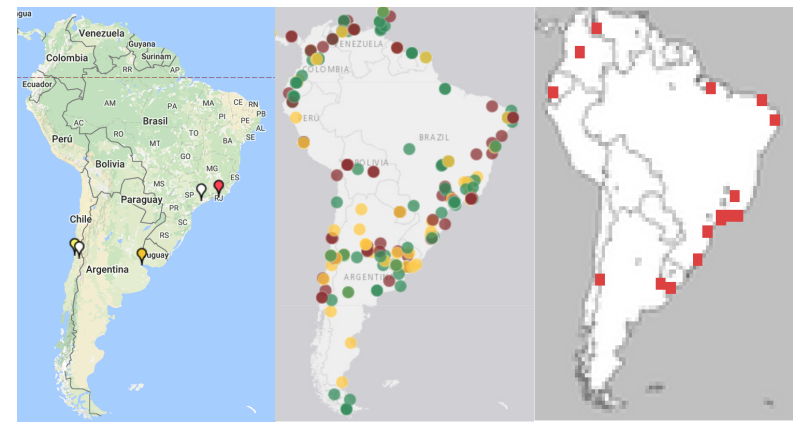

Fig. 2. Ubicación de los puntos de medición de proyectos internacionales en Sudamérica. Acorde la información provista en septiembre de 2016 ArkCAIDA (izquierda) y Planet-Lab (derecha) no cuentan con dispositivos en Bolivia. En cuanto a RIPE Atlas (centro) cuenta con único punto de medición pero fue instalado recién a principios de 2016.

local el tráfico local", los ASes que participan en un PIT no anunciarán los prefijos de los otros miembros por fuera del PIT. De esta manera, la única forma posible de poder atravesar un PIT es desde en uno de sus ASes miembro. Entonces, dado que para poder estudiar la dinámica del PIT se requiere contar con un elemento dentro del PIT, se investigó si existían los recursos utilizados previamente en la literatura, tales como Ark-CAIDA, RIPE Atlas o Planet-Lab. Sin embargo, tal como lo muestra la Figura 2, en Bolivia no se encuentran disponibles estos recursos, salvo una sonda de RIPE Atlas, la cual fue recién instalada en 2016. Otra alternativa hubiera sido utilizar LGs tal como Augustin et al. [11], sin embargo, aunque en www.traceroute.org se anuncie la presencia de este servicio en Bolivia, actualmente el servidor no se encuentra disponible. Por este motivo, frente a la ausencia de recursos para la exploración de la red boliviana de Internet, la alternativa que surgió fue desarrollar una plataforma propia, bautizada Plataforma de Medición Distribuida (PladMeD), la cual contaría con múltiples puntos de observación en diferentes ASes bolivianos.

Disponiendo puntos de observación locales por medio de PladMeD, el análisis de la influencia del PIT en la red local fue generado a través de técnicas conocidas como tomografía de la red [20]. Este paradigma de análisis de Internet se basa en la analogía médica, en donde ante el desconocimiento del sistema, en nuestro caso la interconexión de ASes mientras que en la medicina el estado del cuerpo, se generan pequeños estímulos poco invasivos, de manera tal de recopilar el estado de sistema a través de sus respuestas. En el caso propio de la tomografía de Internet, los estímulos son traceroutes, a través de los cuales se puede reconstruir el mapa de proveedores. Aunque al ejecutar traceroutes se inyectar tráfico en la red, la carga introducida es despreciable con respecto al resto de las aplicaciones.

La arquitectura de PladMeD fue pensada para contar con 2 puntos de observación ubicados en cada uno de los 6 ISPs fundadores del PIT, sin embargo, este objetivo no se pudo alcanzar y se instalaron únicamente dos sondas. En caso de haber contado con la totalidad de las sondas se hubiera podido alcanzar una visión más detallada de las políticas de ruteo de los operadores nacionales, como así también se hubiera contado con más información acerca de la latencia. 
Sin embargo, trabajos previos indican que con dos sondas es posible relevar con gran precisión información de las rutas, y características de la red en las adyacencias de la sonda [21].

Para la implementación de los nodos de PladMeD se dispuso de de Raspberry Pis ${ }^{9} 1$ modelo B, utilizando sistema operativo Raspbian, provistas de scamper [22] para ejecutar los traceroutes necesarios. Los nodos que finalmente fueron instalados se colocaron en ENTEL (AS6568), uno en la Ciudad de la Paz y el otro en Santa Cruz de la Sierra. El nodo de La Paz fue instalado a través de una red de acceso cableada mientras que el nodo de Santa Cruz de la Sierra disponía de un acceso a través de redes LTE. La variación en el tipo de redes de acceso de los nodos se debe a la oferta de acceso disponible en cada ciudad del país. Esta heterogeneidad también es necesaria para poder percibir las diferencias en la calidad de la prestación según la clase de servicio que se ofrece, así generándose un análisis global.

Habiendo presentado la arquitectura de PladMeD es necesario remarcar porqué se descartaron otras alternativas posibles. Ante la ausencia de proyectos de medición de escala global como RIPE Atlas y Ark CAIDA, y dado que se requería desplegar una plataforma, se priorizó generar una plataforma propia, la cual brindara la flexibilidad suficiente para correr rutinas específicas para el estudio del PIT Bolivia. Sin embargo, la instalación de nodos de Planet-Lab en Bolivia hubiera brindado la misma flexibilidad, a través de una plataforma ampliamente difundida en la comunidad científica, y a su vez, permitiendo a otros investigadores hacer uso del recurso. No obstante, la instalación de PlanetLab hubiera conducido a dos importantes desventajas con respecto a la plataforma creada a través de PladMeD. En primer lugar, los nodos de Planet-Lab deben ser instalados en redes académicas, por lo cual los puntos de medición no brindarían un fiel reflejo del impacto del PIT en los usuarios residenciales. En segundo lugar, el costo que habría implicado comprar e instalar servidores, los cuales fueran capaces de brindar el servicio de Planet-Lab, hubiera incrementado sustancialmente el costo del proyecto.

Para poder conseguir un panorama de la red de Bolivia, PladMeD ejecutó traceroutes hacia un subconjunto de los prefijos asignados por LACNIC a Bolivia. En Bolivia, al igual que en el resto de los países, operan ASes extranjeros, cuyo prefijos puede haber sido solicitados ante otro RIR ${ }^{10}$. Sin embargo, la cantidad de operadores extranjeros en Bolivia es sumamente baja, incluso menor que en el resto de los países de la región. Además, la baja presencia de operadores extranjeros hará poco probable que direcciones asignadas a estos ISPs en Bolivia sean realmente operadas en el exterior. Otro caso a considerar, pero de todas maneras improbable, es que los proveedores de capital nacional dispongan de recursos ubicados en el extranjero. Bajo todas estas hipótesis, y aunque no sea una forma completamente certera, esta metodología sería suficientemente representativa. La elección del subconjunto de direcciones bolivianas se generó bajo la metodología "IPv4 routed /24 topology", por la cual se dividen todos los prefijos a analizar en redes de tamaño Clase "C" y luego se selecciona una dirección

\footnotetext{
${ }^{9}$ Raspberry Pi es una microcomputadora de bajo costo capaz de correr sistemas operativos Linux. https://www.raspberrypi.org

${ }^{10}$ RIR: Regional Internet registry.
}

\begin{tabular}{|c|c|c|}
\hline Categoría & PIT & Nacional \\
\hline \hline IXP & 1 & 1 \\
\hline P2P & 0 & 1 \\
\hline Anómalas & 1 & 0 \\
\hline Internacionales & 0 & 0 \\
\hline
\end{tabular}

TABLE I

TABLA DE CLASIFICACIÓN DE LOS TRACEROUTES ENTRE USUARIOS LOCALES SEGÚN EL MÉTODO DE DISTRIBUCIÓN. LA COLUMNA "PIT" INDICA SI EL TRACEROUTE CONTIENE LOS PREFIJOS DEL PIT BOLIVIA, MIENTRAS QUE LA COLUMNA "NACIONAL" AFIRMA SI LA TOTALIDAD DE LOS PREFIJOS OBSERVADOS PERTENECE A ASES BOLIVIANOS.

objetivo dentro de cada subconjunto [23].

La campaña de exploración de Bolivia se generó enviando traceroutes durante 20 horas por días, todos los días. Aunque se podría haber extendido la campaña de medición durante toda la noche, los resultados hubieran sido francamente similar a causa de la merma en el volumen de tráfico. Por este motivo, se han dejado cuatro horas libres para sincronizar las sondas con el nodo central, hacer la transferencia de las mediciones y también eventuales tareas de mantenimiento. Acorde con la metodología de selección de destinos, la densidad de traceroutes por hora promedio fue de 4400. Mediante esta tasa de muestreo se pudo analizar en detalle las características de las red, y la naturaleza de sus variaciones. A su vez, todo la información recolectada fue enviada a una unidad de procesamiento central, en la cual se confeccionaron estadísticas con la información agregada, de forma tal de poder analizar la evolución de la red.

\section{Resultados OBTENidos}

Por medio de los datos recopilados por PladMeD se ha analizado la evolución semanal de parámetros de la red susceptibles ante la presencia de un PIT, durante los primeros seis meses del de funcionamiento, lo cual abarcó entre junio de 2014 y enero de 2015. Para analizar el impacto del PIT en la infraestructura nacional y en la mejora de la calidad de la experiencia de usuario se ha monitoreado la evolución de dos parámetros frecuentemente utilizados por los operadores de la red, tales como la distancia en número de saltos y la latencia. A su vez, se han definido dos nuevos parámetros, llamados tiempo accesible y rutas locales, de manera tal de comprender aún más los efectos del PIT. A través de tiempo accesible se pretende determinar que porcentaje de los traceroutes enviados utilizaron el PIT y por medio de rutas locales el porcentaje de las rutas observadas, entendida una ruta como una secuencia de direcciones IP, fueron enviadas por el PIT.

En cuanto a la granularidad del análisis, se eligió una semana, ya que tomando intervalos diarios se hubieran detectado fluctuaciones propias del comportamiento social, como por ejemplo la variación semana fin de semana. De manera opuesta, si se hubiera elegido un análisis mensual, la granularidad hubiera ocultado ciertos fenómenos propios de inestabilidades temporales. Finalmente se optó por utilizar intervalos semanales, para poder analizar la tendencia sin tener que reparar en fenómenos aislados de días excepcionales, como feriados y fines de semana.

Los cuatros parámetros bajo estudio fueron a su vez clasificados en cuatro categorías dependiendo el método de transporte de los paquetes, tal como se observa en 

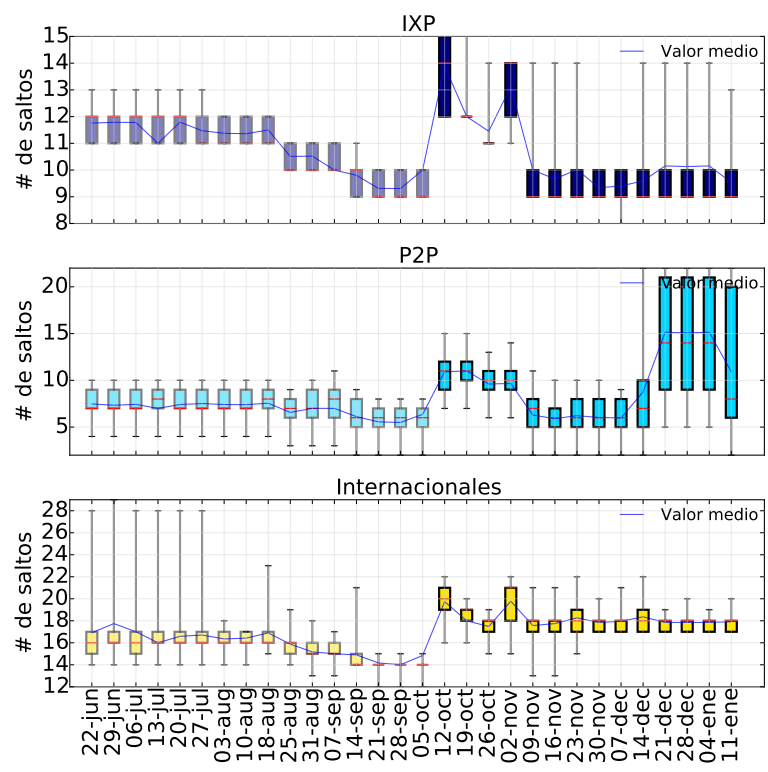

Fig. 3. Evolución del número de hops entre origen y destinos locales, clasificado en tres categorías dependiendo la forma de distribución. En los boxplots se han usado dos colores para diferenciar desde donde se realizaron las exploraciones. Los colores más claros corresponden a las mediciones ejectuadas desde la Ciudad de La Paz y los más oscuros a las mediciones desde Santa Cruz de la Sierra, ambas desde Entel (AS6568).

el Cuadro I. Bajo categoría IXP se encuentran todos los traceroutes que contienen a la red del PIT y todos sus saltos contienen direcciones IP asignadas a Bolivia. La categoría P2P determina que traceroute fueron enviados a través de vínculos entre pares privados, por lo cual no utilizaron el PIT pero nunca abandonaron la nación. El rótulo Internacionales fue utilizando para aquellos paquetes que deben atravesar AS extranjeros para poder alcanzar un destino en Bolivia, por lo tanto en los traceroutes no aparecen direcciones de PIT pero se observan direcciones extranjeras. La última clasificación es Anómalas que son aquellos paquetes que sí han atravesado el PIT pero además sus traceroutes contienen direcciones extranjeras.

Este método de clasificación se encuentra estrechamente relacionado en la capacidad de discernir si una dirección IP es nacional. La metodología se basó en determinar si los prefijos pertenecían a los delegados por LACNIC a Bolivia, y tomando las direcciones privadas como carentes de información. Se podrían haber utilizado bases de datos de geolocalización, no obstante su precisión ha sido debatida en numerosas oportunidades [24]. Sin embargo, la confiabilidad de nuestro método radica en la baja probabilidad de encontrar una dirección IP asignada a Bolivia fuera de este país. A su vez, la presencia de ISPs extranjeros en Bolivia es baja y los que sí se encuentran radicados en el país operan bajo empresas nacionales.

Aunque no se esperaban a priori observar resultados para la categoría Anómala, las mediciones mostraron la presencia de alguna de estas rutas a lo largo del período. Una de las causas podría un error a la hora de la configuración de las tablas de ruteo. Sin embargo, otra hipótesis plausible es que este comportamiento se haya debido a un secuestro de rutas [25], escenario en el cual algún ISP utilizó y difundió a través de BGP prefijos de una red de Bolivia sin autorización
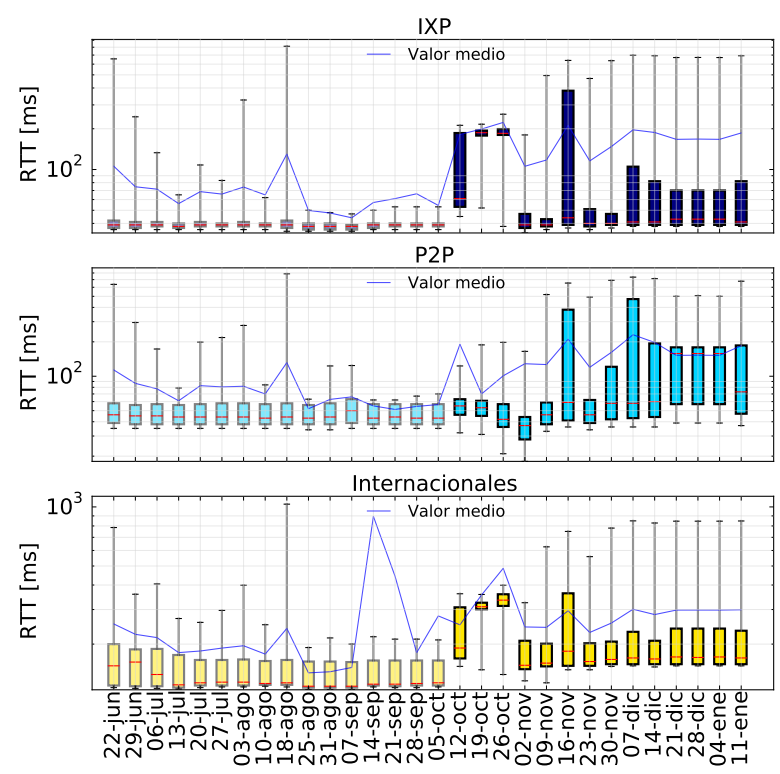

Fig. 4. Evolución de la latencia entre orígenes y destinos locales, clasificado en tres categorías dependiendo la forma de distribución. En este caso el mínimo y el máximo corresponden a los percentiles 5\% y $95 \%$ respectivamente. En los boxplots se han usado dos colores para diferenciar desde donde se realizaron las exploraciones. El tonos más claros corresponde a mediciones ejectuadas desde la Ciudad de La Paz y en tonos más oscuros desde Santa Cruz de la Sierra, ambas desde Entel (AS6568). La semana del 18 de agosto muestra un marcado creciemiento del valor máximo producto del $512 k$ day.

o conocimiento de sus propietarios.

Finalmente, con la información recopilada de la campaña de medición se pudieron confeccionar mapas de la topología de Bolivia, observado los efectos del PIT y de la presencia de puntos de observación en sus inmediaciones.

A fines de lograr difundir los resultados, toda la información procesada esta disponible en http://cnet.fi.uba.ar/ PIT.

\section{A. Mediciones de infraestructura y calidad}

Las Figuras 3 y 4 muestra la evolución semanal de la cantidad de saltos y la latencia entre origen y destino nacional, diferenciado para cada una de las categorías exceptuando Anómalas. En ellas se presentan boxplots para poder mostrar la dispersión en los valores observados como así también el valor medio en línea solida. En la secuencia de boxplot se puede observar dos tonos de colores en la cajas, donde las cajas más claras fueron creadas utilizando los datos recolectados por el nodo de la Ciudad de La Paz, en el proveedor Entel (AS6568), mientras las más oscuras corresponden a mediciones efectuadas desde el mismo proveedor pero con una sonda ubicada en Santa Cruz de la Sierra.

A pesar de no contar con mediciones previas a la implementación del PIT, se puede suponer que previo a su creación, el envió de los paquetes entre proveedores locales se realizaba principalmente a través de proveedores internacionales. De esta manera, al observar la evolución de la distancia presentada en la Figura 3 se aprecia una diferencia de $50 \%$ entre las categorías IXP e Internacionales cuando se mide desde La Paz, y una diferencia más pronunciada cuando se cambia al punto de observación fuera de la capital boliviana. Aunque las mediciones hayan comenzado luego 
de instalado el PIT, el periodo de la campaña de medición coincidió con los primeros meses, donde todavía los operadores se encontraban en fase de optimización. Dado que la cantidad de saltos es una medida discreta, sería inusual encontrar grandes variaciones. Sin embargo, la secuencia de La Paz muestra una leve tendencia decreciente. Comparando el efecto en cada una de las categorías luego de cambiar el punto de observación, tanto P2P como Internacionales sufren severos cambios y aumento de la variabilidad al pasar a Santa Cruz. Al contrario, la categoría IXP se muestra constante en valor mediano y distancia intercuartil durante todo el período, lo que muestra una calidad de acceso constante a la redes nacionales sin tener mayor impacto desde el punto donde se esté accediendo.

En la Figura 4 se muestra la evolución de la latencia a lo largo del período de observación para las tres categorías. Aunque los gráficos de la latencia presentan una tendencia similar a los de la cantidad de saltos, el análisis de los datos obtenidos será más pormenorizado debido a que la latencia es un parámetro fundamental en la calidad de la experiencia. Es importante remarcar que producto de marcada dispersión de los valores máximos, los boxplot de la latencia han sido generados tomando el mínimo y máximo como los percentiles 5\% y $95 \%$ respectivamente. En primer lugar, los gráficos de IXP y P2P muestran un latencia mediana de alrededor de 40ms, mientras que la categoría Internacionales muestra una valor mediano alrededor de los 160ms. Utilizando la hipótesis de que el PIT reemplazó rutas que anteriormente se enviaban al extranjero, la ventaja de la instalación del PIT muestra una reducción del $75 \%$ del valor Internacional presentando significativas ventajas. Más aún la interconexión a través del PIT muestra que se obtienen valores idénticos a los observados en $\mathrm{P} 2 \mathrm{P}$ pero con la ventaja de poder alcanzar mayor cantidad de destinos.

Deteniéndonos en la evolución de la gráfica se observa un aumento considerable de la distancia intercuartil una vez que se incluye la sonda ubicada en Santa Cruz de la Sierra. Aunque esto ya había sido observado en la cantidad de hops, aquí también se pone de manifiesto en la latencia. El aumento en la dispersión la incluir las mediciones de Santa Cruz puede ser formulado a través de múltiples hipótesis. En primer lugar, la dispersión es independiente de las categorías, por lo cual se puede presuponer que la causa, no está a nivel de capa de red, sino que por debajo en el nivel de enlace. En la latencia esto se puede manifestar como congestión de la red de acceso, ya que la sonda de Santa Cruz de la Sierra se encontraba conectada a través de la red LTE. En el caso del aumento de la cantidad de saltos, el uso de la infraestructura móvil también puede haber conducido al alejamiento con respecto a algunos destinos. En segundo lugar, es necesario remarcar que La Paz tiene un rol central en la distribución de los datagramas IP, por lo cual los envíos desde Santa Cruz tienen que ser enviados hacia La Paz. Este escenario hace que los paquetes recorran mayor cantidad de saltos, y probablemente atraviesen enlaces congestionados, lo que nuevamente sería una hipótesis valida para explicar esta marcada variabilidad.

El valor medio se encuentra estable para todos los gráficos excepto un pico específico presente en Internacionales la semana del 14 de septiembre. Sin embargo, la latencia tiene un
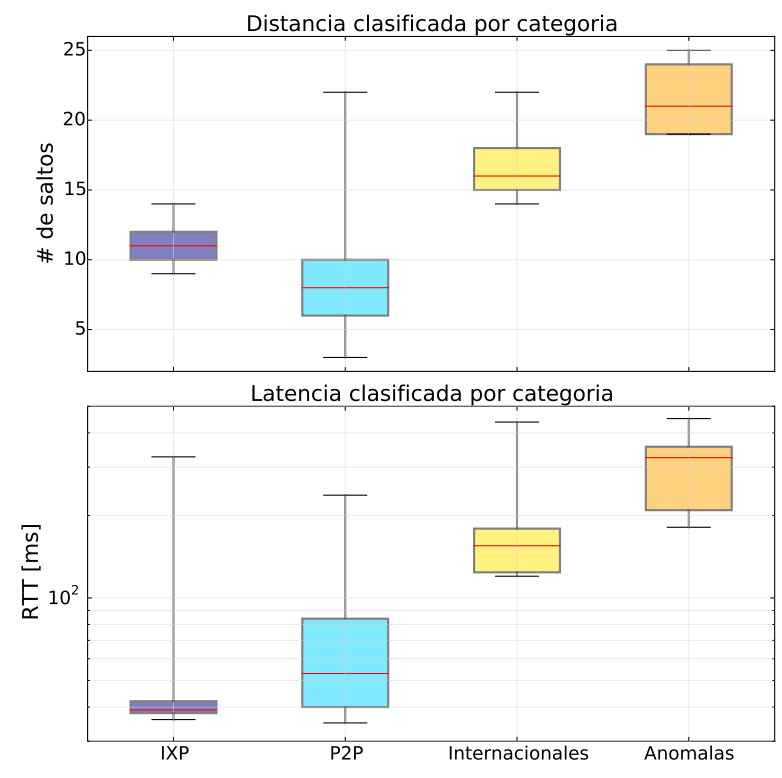

Fig. 5. Comparación de la distancia entre cantidad de saltos y latencia por categoría, agregando todos los datos recopilados durante los meses de exploración.

patrón de comportamiento de cola pesada y el valor medio tiene alta variabilidad ante esta clase de distribuciones. Al no ver cambios significativos, en el boxplot correspondiente a esta semana, podemos atribuir su variación a un par de mediciones fuera de escala.

Aunque el PIT esté destinado a generar interconexión entre ASes locales, y los prefijos anunciadas aquí pertenecen única o principalmente a sus miembros, no se encuentra exento a poder ser afectado por inestabilidades en el sistema de ruteo global. Durante la semana del 18 de agosto de 2014 sucedió un fenómeno conocido como el " $512 k$ day" 11 , en el cual se superó dicha cantidad de entradas en las tablas BGP, lo cual desencadenó un efecto de inestabilidad de la red mundial debido a que gran parte de los routers no pueden operar con más de $512 \mathrm{k}$ prefijos. Como consecuencia, aquellos routers que vieron excedida su memoria comenzaron a sobreescribir aleatoriamente ciertas entradas, dejando inaccesibles aquellos prefijos eliminados para darle lugar a los nuevos. Este fenómeno se puso de manifiesto en la infraestructura local, en particular en la latencia mostrando un crecimiento del valor máximo de la latencia en las tres categorías. Sin importar que el origen y destino fuera nacional, la inestabilidad del sistema de ruteo generó un marcado incremento de este percentil aún en rutas nacionales.

Para hacer una comparación general de cada una de las categorías, la Figura 5 muestra los boxplots de la distancia en hops y la latencia durante todo el período bajo análisis. En primer lugar, en la distancia se puede observar una notable reducción de hops al pasar de la categoría Internacional a IXP, obteniendo valores similares a los de P2P. A su vez, se muestra como ante la presencia de rutas anómalas, ya sea por errores o secuestro, los valores percibidos fueron incluso por encima de los internacionales. Con respecto a la latencia, la recolección de datos de PladMeD mostró que la categoría

\footnotetext{
${ }^{11}$ 512k day: https://en.wikipedia.org/wiki/Border \_Gateway \_Protocol
} 


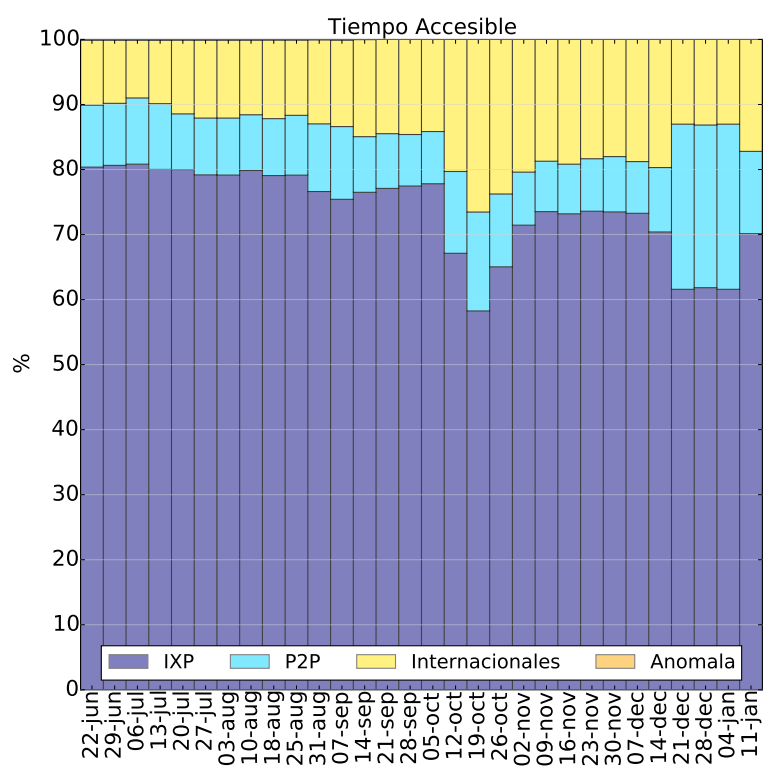

Fig. 6. Evolución del Tiempo Accesible a lo largo del periodo de observación. Este parámetro indica la distribución del tráfico generado para el relevamiento, donde las categorías son los métodos de distribución.

IXP mostró los valores más bajos, seguido por P2P y luego por Internacionales. Para estos casos la mediana fue $39 \mathrm{~ms}$, $53 \mathrm{~ms}$ y $155 \mathrm{~ms}$ respectivamente. Aunque IXP muestre una leve mejora en su valor mediano respecto $\mathrm{P} 2 \mathrm{P}$, su ventaja es incluso más pronunciada cuando se tiene en cuenta la distancia intercuartil. No obstante, durante el lapso medido la utilización de las capacidades de PIT eran sumamente bajas, por lo cual la baja dispersión se puede debe a la ausencia de congestión.

Las Figuras 6 y 7 muestran la incidencia del PIT sobre la forma de distribución del tráfico nacional. En particular la Figura 6 mide qué porcentaje de las mediciones fueron enviadas por cada sistema, mostrando que a lo largo del tiempo el PIT (categoría IXP) distribuye entre el $60 \%$ y $80 \%$ de los paquetes generados con origen y destino nacional. Suponiendo la hipótesis que el PIT creó un nuevo punto de intercambio local para reemplazar rutas que dependían de proveedores en el extranjero, y no relego rutas de interconexión a través de enlaces entre pares, la medición de P2P de $10 \%$ muestra cual era el grado de interconexión nacional antes de la creación de PIT. Aunque la categoría Anómala se encuentre presente en la leyenda, sólo durante algunas semanas se detectó este comportamiento, y su participación en el total de las rutas analizadas nunca superó el $0.13 \%$, por lo cual es casi imperceptible. En cuanto a la Figura 7, ésta hace referencia a la proporción de rutas enviadas a través del PIT por semana. Esto significa que con que al menos una ruta haya pasado por el PIT una única vez, es suficiente para contabilizarla. Si la forma de distribución varía de categoría a lo largo de la semana hará que la suma de los conjuntos superé el $100 \%$. Al igual que en Tiempo Accesible, las rutas locales muestran que entre el $60 \%$ y $80 \%$ de los destinos fueron alcanzados al menos una vez a través del PIT durante esa semana. No obstante, esta medición muestra un valor más elevado en la categoría Internacional, lo que significa que hubo destino alcanzados a través del

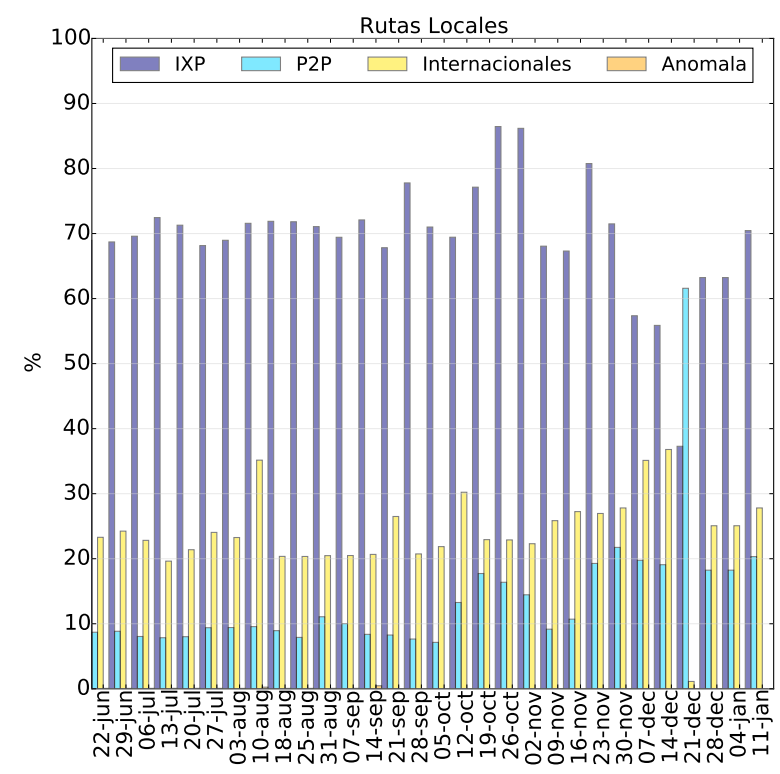

Fig. 7. Distribución del parámetros Rutas Locales a lo largo del período bajo análisis. En este gráfico se muestra el porcentaje de destinos alcanzado a través de los distintos métodos de distribución durante una semana. Dado que las rutas pueden variar, durante una semana el destino puede haber sido alcanzado por dos sistemas, generando que la suma de las categorías pueda exceder el $100 \%$.

PIT y de proveedores extranjeros durante la misma semana.

\section{B. Relevamiento de la topología y su robustez}

Continuando con el trabajo realizado por Silva Berenguer et al. [18], se buscó generar mapas de la topología de la red boliviana que contaran con mayor precisión que los obtenidos a través de proyectos internacionales. Con esta finalidad, en primer lugar, se recopilo información BPG provista por RIPE RIS (RR) y RouteViews (RV), tomando únicamente los Sistemas Autónomos que tuvieran presencia en Bolivia de acuerdo con RIPEstat Data API. El resultado de este procedimiento dio lugar a la posibilidad de crear grafo de interconexión, el cual debido a la característica de los puntos de observación, carecía de información local. Luego, se agregó los datos de interconexión recopilados de la exploración de PladMeD y se compararon las diferencias al agregar la información local.

En la Figura 8 se han construido dos mapas de interconexión mostrando las diferencias del grafo al agregar la información local. Dado que los puntos de observación de RIPE RIS y RouteViews, no se encuentran en las adyacencias del PIT y que los ASes que componen el PIT no propagan estos prefijos hacia el exterior, no es posible ver el aumento de la interconexión local a través de estos mapas BGP. Al agregar la información proveniente de PladMeD, aumenta la cantidad de enlaces nacionales, el grado de todos los ASes y la estructura de la red. El mayor aumento en el grado se observa en Entel (AS6568), y aunque las mediciones hayan sido efectuadas desde aquí, esto se debe a que el PIT está alojado en este AS. También al agregar la información local se muestra un marcado posicionamiento de DiViNetworks (AS57731) como el nodo de mayor grado, funcionando como principal proveedor de tránsito nacional.

En ambos grafos presentes en la Figura 8 se observa la presencia de un subgrafo formado por tres ASes, los 


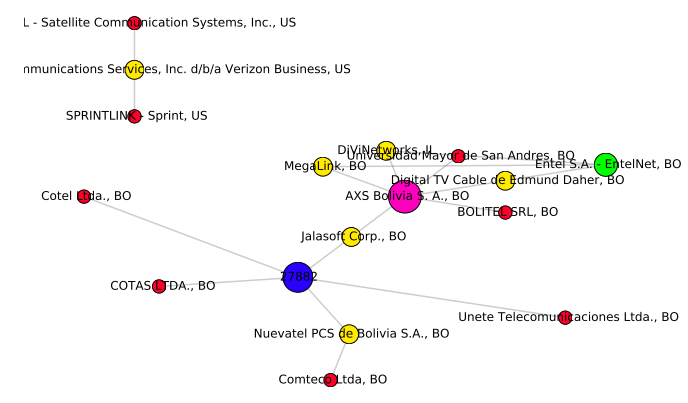

(a) Utilizando RR y RV

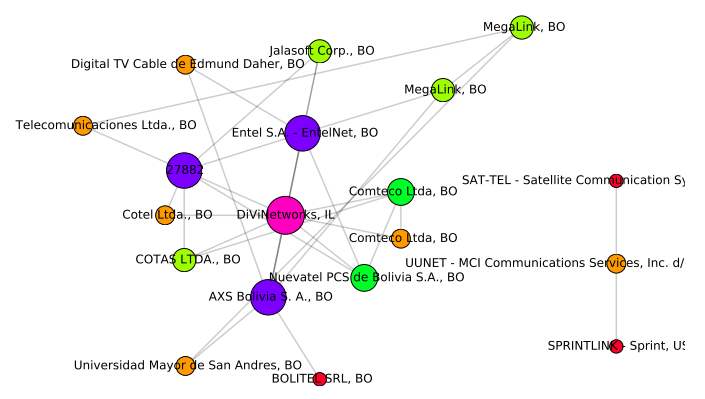

(b) Utilizando RR, RV y PladMeD

Fig. 8. Comparación de grafos de interconexión en Bolivia al agregar información local. En la Figura 8a se construyó el grafo utilizando información de RR y RV, mientras que en la Figura 8b se agregaron los vínculos observados a través de PladMeD. Los colores y los tamaños de los nodos dependen la cantidad de enlaces de cada AS.

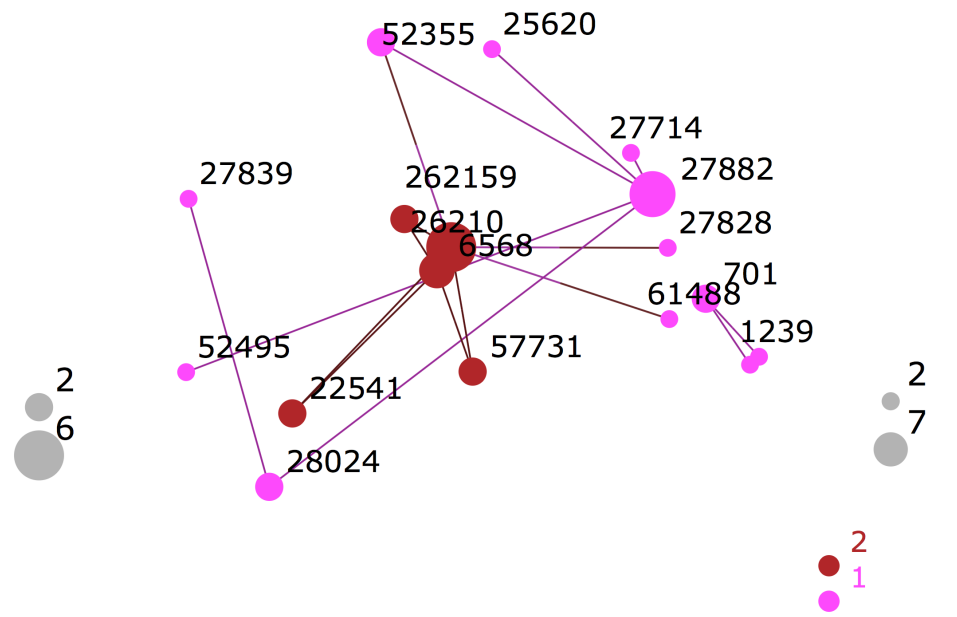

(a) Utilizando RR y RV

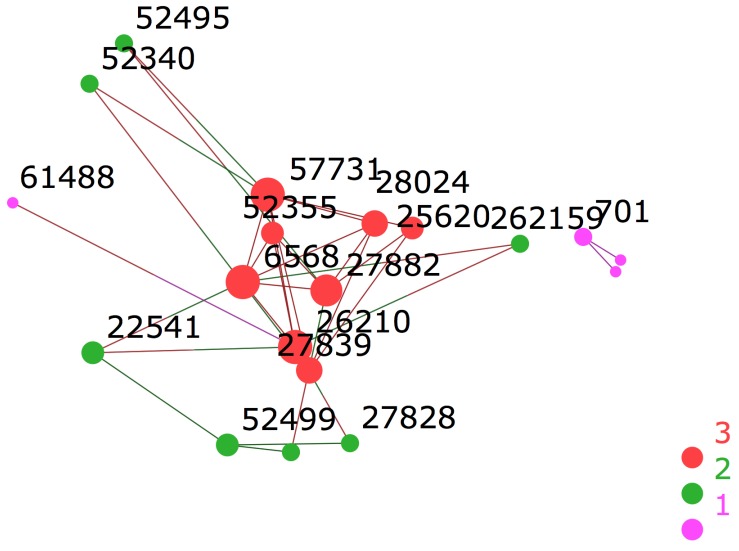

(b) Utilizando RR, RV y PladMeD

Fig. 9. Comparación de la descomposición en $k$-núcleos del grafo de interconexión en Bolivia. En la Figura 9a se construyó el grafo utilizando información de RR y RV, mientras que en la Figura 9b se agregaron los vínculos observados a través de PladMeD. Los colores varían según el máximo núcleo al que el AS pertenece. El tamaño de los nodos dependen la cantidad de enlaces de cada AS.

cuales no se encuentran conectados al resto del grafo. Este subgrafo aislado esta compuesto por Sprint (AS1239), Verizon (AS701) y Satellite Communication (AS10434). Aunque RIPEstat Data API indique que tienen presencia en Bolivia, a través de PladMeD no pudimos detectar su interconexión con ASes nacionales.

La Figura 9 muestra el grafo de la red boliviana obtenido con LaNet-vi [26], el cual utiliza la descomposición en $k$-núcleos. La utilización de k-cores sirve para analizar la robustez de la red. Esto viene dado a partir de la definición de $k$-núcleo que enuncia que un $k$-núcleo es el subgrafo inducido máximal en el cual todos los nodos tiene grado al menos k. En le gráfico se indica el grado del AS a través del diámetro de la esfera, y la escala de colores refleja la pertenencia de los ASes a los distintos $k$-núcleos.

Los resultados de la descomposición en $k$-núcleos releva los niveles de jerarquía, y además, en este caso en particular, se puede verificar si el grafo es núcleo-conexo. Esta última propiedad está relacionada con la robutez, es decir el número de caminos disjuntos que unen dos ASes cualquiera. En particular dado dos nodos que pertenecen al núcleo $k$, existen $k$ caminos disjuntos entre estos dos cuando la red núcleo-conexa. Cabe señalar que un AS que se encuentra en el núcleo $k$ también se encuentra en los núcleo inferiores.

La comparación de ambos grafos revela, que es necesario contar con información local para poder tener una noción más clara de la estructura de la red. En particular, en secciones anteriores se mencionó que el proyecto sólo contó con dos sondas de medición, y esto fue suficiente para aportar nuevas aristas que incrementaron el $k$-núcleo máximo. Aquí la en la descomposición en $k$-núcleos presente en la Figura 9 aporta un grado de información extra con respecto a la Figura 8, ya que en esta última no se puede cuantificar la resiliencia de la red.

\section{Conclusiones}

La exploración de la red de Bolivia dejó de manifiesto la potencialidad y las ventajas que provee un PIT en la inter- 
conexión local. Los resultados de la campaña de medición mostraron la evolución de la calidad de la experiencia de los usuarios al utilizar formas de interconexión locales en lugar de enlaces internacionales. A su vez, en el análisis de los grafos se ha mostrado como al crear un PIT se incrementa la confiabilidad y robustez de la red nacional.

La campaña de medición no mostró únicamente la naturaleza de la red boliviana, sino que también manifestó el grado de inexactitud de los proyectos de relevamiento de la topología de Internet en esta región.

Sin embargo, los cambios observados en la tomografía del PIT también tendrán un impacto económico. La reducción del tráfico internacional observado a través de los parámetros Tiempo Accesible y Rutas Locales, generará un marcado beneficio económico para los ISPs. A su vez, la mejora de infraestructura de la red nacional podrá ser un factor determinante para la proliferación de nuevos servicios y oportunidades comerciales a través de Internet.

Por último, quedará como trabajo futuro estudiar el acceso de los usuario nacionales a las CDNs y los cambios significativos que puede llegar a presentarse en el eventual caso de que alguna CDN se instale en el PIT.

\section{Agradecimientos}

La realización de este trabajo fue producto del trabajo y la voluntad de una gran cantidad de personas, quienes compartieron junto el autor el interés y la necesidad de estudiar la interconexión en Bolivia. Por este motivo, merecen un especial reconocimiento los miembros de la Autoridad Telecomunicaciones y Transportes de Bolivia como así también a la institución. Dentro de los miembros de la ATT debo destacar especialmente a Carlos Sanabria quien coordino en proyecto en Bolivia.

Por otra parte, se agradece el apoyo de Internet Society, organización que fomentó la instalación de PIT y a su vez, apoyo la necesidad de llevar adelante un estudio de su evolución. En particular quiero remarcar a Christian O'Flaherty, quien como miembro de Internet Society, fue el encargado de que el PIT se transformara en un proyecto viable, y a su vez, nos proporcionó todo su apoyo para realizar este proyecto académico.

Finalmente, se agradece la colaboración académica de colegas Hernán Galperín y Sofía Silva Berenguer que trabajaron junto a nosotros y ayudaron a enriquecer este proyecto, aportando cada uno su punto de vista como también largas horas de dedicación.

En carácter de autor del artículo agradezco al CONICET por el otorgamiento de la Beca Interna Doctoral.

\section{REFERENCES}

[1] Chatzis, Nikolaos and Smaragdakis, Georgios and Feldmann, Anja and Willinger, Walter, "There is more to IXPs than meets the eye," ACM SIGCOMM Computer Communication Review, vol. 43, no. 5, pp. 19-28, 2013.

[2] International Telecommunication Union, "World Telecommunication Policy Forum-Internet Exchange Points," https://www.itu.int/en/wtpf13/Documents/backgrounder-wtpf-13-ixps-en.pdf, ITU, 2013.

[3] G. Pallis and A. Vakali, "Insight and perspectives for content delivery networks," Communications of the ACM, vol. 49, no. 1, pp. 101-106, 2006.

[4] Dhamdhere, Amogh and Dovrolis, Constantine, "The Internet is flat: modeling the transition from a transit hierarchy to a peering mesh,' in Proceedings of the 6th International Conference. ACM, 2010, p. 21.
[5] Labovitz, Craig and Iekel-Johnson, Scott and McPherson, Danny and Oberheide, Jon and Jahanian, Farnam, "Internet inter-domain traffic," in ACM SIGCOMM Computer Communication Review.

[6] K. Xu, Z. Duan, Z.-L. Zhang, and J. Chandrashekar, "On properties of internet exchange points and their impact on as topology and relationship," in NETWORKING 2004. Networking Technologies, Services, and Protocols; Performance of Computer and Communication Networks; Mobile and Wireless Communications. Springer, 2004, pp. 284-295.

[7] D. McRobb, K. Claffy, and T. Monk, "Skitter: Caida?s macroscopic internet topology discovery and tracking tool, 1999."

[8] N. T. Spring, D. Wetherall, and T. E. Anderson, "Scriptroute: A public internet measurement facility." in USENIX Symposium on Internet Technologies and Systems, 2003.

[9] R. Views, "University of oregon route views project," http://www.routeviews.org/, University of Oregon, 2000.

[10] N. RIPE, "RIS: Routing Information Service," 2006.

[11] B. Augustin, B. Krishnamurthy, and W. Willinger, "IXPs: mapped?" in Proceedings of the 9th ACM SIGCOMM conference on Internet measurement conference. ACM, 2009, pp. 336-349.

[12] B. Ager, N. Chatzis, A. Feldmann, N. Sarrar, S. Uhlig, and W. Willinger, "Anatomy of a large european IXP," in Proceedings of the ACM SIGCOMM 2012 conference on Applications, technologies, architectures, and protocols for computer communication. ACM, 2012, pp. 163-174

[13] J. C. Cardona Restrepo and R. Stanojevic, "A history of an internet exchange point," ACM SIGCOMM Computer Communication Review, vol. 42, no. 2, pp. 58-64, 2012.

[14] A. Gupta, M. Calder, N. Feamster, M. Chetty, E. Calandro, and E. Katz-Bassett, "Peering at the internet frontier: A first look at isp interconnectivity in africa," in Passive and Active Measurement. Springer, 2014, pp. 204-213.

[15] R. Fanou, P. Francois, and E. Aben, "On the diversity of interdomain routing in africa," in Passive and Active Measurement. Springer, 2015, pp. 41-54.

[16] Georgia Tech, Princeton University and M-Lab, "BISMark: Broadband Internet Service Benchmark," http://projectbismark.net/, 2010.

[17] RIPE, NCC, "RIPE Atlas," https://atlas.ripe.net, 2010.

[18] S. S. Berenguer, E. Carisimo, J. I. Alvarez-Hamelin, and F. V. Pintor, "Hidden internet topologies info: Truth or myth?" in Proceedings of the 2016 workshop on Fostering Latin-American Research in Data Communication Networks. ACM, 2016, pp. 4-6.

[19] P. Gill, M. Arlitt, Z. Li, and A. Mahanti, The Flattening Internet Topology: Natural Evolution, Unsightly Barnacles or Contrived Collapse? Berlin, Heidelberg: Springer Berlin Heidelberg, 2008, pp. 1-10. [Online]. Available: \{http://dx.doi.org/10.1007/ 978-3-540-79232-1_1\}

[20] k. claffy, T. Monk, and D. McRobb, "Internet Tomography," Nature, Jan 1999.

[21] L. Dall'Asta, J. I. Alvarez-Hamelin, A. Barrat, A. Vázquez, and A. Vespignani, "Exploring networks with traceroute-like probes: Theory and simulations." Theor. Comput. Sci., vol. 355, no. 1, pp. 6-24, 2006.

[22] Luckie, Matthew, "Scamper: a scalable and extensible packet prober for active measurement of the internet," in Proceedings of the 10th ACM SIGCOMM conference on Internet measurement. ACM, 2010, pp. 239-245.

[23] Claffy, Kimberly and Hyun, Young and Keys, Ken and Fomenkov, Marina and Krioukov, Dmitri, "Internet mapping: from art to science," in Conference For Homeland Security, 2009. CATCH'09. Cybersecurity Applications \& Technology.

[24] Poese, Ingmar and Uhlig, Steve and Kaafar, Mohamed Ali and Donnet, Benoit and Gueye, Bamba, "IP geolocation databases: Unreliable?" ACM SIGCOMM Computer Communication Review, vol. 41, no. 2, pp. 53-56, 2011

[25] M. Wählisch, O. Maennel, and T. C. Schmidt, "Towards detecting bgp route hijacking using the rpki," ACM SIGCOMM Computer Communication Review, vol. 42, no. 4, pp. 103-104, 2012.

[26] Mariano G. Beiró and J. Ignacio Alvarez-Hamelin and Jorge R. Busch, "A low complexity visualization tool that helps to perform complex systems analysis," New J. Phys, vol. 10, no. 12, p. 125003, 2008. [Online]. Available: http://dx.doi.org/10.1088/1367-2630/10/ $12 / 125003$ 